\title{
Using typing techniques in a specific outbreak: the ethical reflection of public health professionals
}

\author{
B. RUMP ${ }^{1,2}$, C. CORNELIS ${ }^{3}$, F. WOONINK ${ }^{1}$, J. VAN STEENBERGEN ${ }^{2,4}$, \\ M. VERWEIJ ${ }^{5}$ AND M. HULSCHER ${ }^{6}$ \\ ${ }^{1}$ Regional Health Service Utrecht region (GGDrU), Zeist, The Netherlands \\ ${ }^{2}$ Centre for Infectious Disease Control, National Institute of Public Health and the Environment (RIVM), \\ Bilthoven. The Netherlands \\ ${ }^{3}$ Ethics Institute, Utrecht University, Utrecht, The Netherlands \\ ${ }_{5}^{4}$ Centre of Infectious Diseases, Leiden University Medical Centre, Leiden, The Netherlands \\ ${ }^{5}$ Section Communication, Philosophy and Technology, Wageningen University, Wageningen, The Netherlands \\ ${ }^{6}$ Scientific Institute for Quality of Healthcare (IQ Healthcare), Radboud University Medical Centre, Nijmegen, \\ The Netherlands
}

Received 24 May 2016; Final revision 7 October 2016; Accepted 8 January 2016;

first published online 13 February 2017

\section{SUMMARY}

Typing techniques are laboratory methods used in outbreak management to investigate the degree to which microbes found within an outbreak are related. Knowledge about relational patterns between microbes benefits outbreak management, but inevitably also tells us something about the relational patterns of the people hosting them. Since the technique is often used without explicit consent of all individuals involved, this may raise ethical questions. The aim of this study was to unravel the complex ethical deliberation of professionals over the use of such techniques. We organised group discussions $(n=3)$ with Dutch outbreak managers $(n=23)$. The topic list was based on previously identified ethical issues and discussions were analysed for recurrent themes. We found that outbreak managers first and foremost reflect on the balance of individual harm with public health benefit. This key question was approached by way of discussing four more specific ethical themes: (1) justification of governmental intervention, (2) responsibility to prevent infections, (3) scientific uncertainty and (4) legal consequences. The themes found in this study, rephrased into accessible questions, represent the shared ethical understanding of professionals and can help to articulate the ethical dimensions of using molecular science in response to infectious disease outbreaks.

Key words: Ethics, molecular epidemiology, outbreaks, public health ethics, typing.

\section{INTRODUCTION}

Even though traditional epidemiological methods are still the cornerstone of outbreak investigation, the use of (molecular) typing methods over the past decade has quickly gained importance $[1,2]$. Typing methods

\footnotetext{
* Author for correspondence: B. O. Rump, Regional Health Service Utrecht region (GGDrU), Postbus 51, 3700 AB, Zeist, The Netherlands.

(Email: brump@ggdru.nl)
}

are laboratory techniques that are used to discriminate between different bacterial isolates of the same species. They can be highly discriminatory and can tell something about the degree to which different microbial isolates share characteristics such as a DNA sequence. If the stability of such a characteristic is known, then the degree to which different microbes found in an outbreak are related can be estimated $[2,3]$. This makes the technique very helpful in attributing or discharging sources to an outbreak [4-7]. 
During the Escherichia coli O104:H4 outbreak in Germany in 2011, for instance, the technique was used to quickly rule out Shiga Toxin-Producing E. coli (STEC) detected on Spanish cucumber as a source and eventually attributed the outbreak to Fenugreek sprouts [8]. In 2012 a large outbreak of Salmonella Thompson was linked to Dutch smoked salmon [9]. As valuable as the technique is for outbreak management, the degree of relatedness between different microbes found in an outbreak inevitably also reveals relational patterns of the individuals hosting them. This ability of molecular techniques to show relational patterns between individuals with more certainty than before, influences the way we conceptualise ethical issues such as privacy, consent and obligation to avoid spreading a disease [10]. Furthermore this inference about the directionality of transmission can easily be perceived by the public as an answer to the 'who infected whom?' question. An answer to this question may be used for other purposes, notably in support of legal or moral claims about responsibility and liability [3, 10-13].

Success of future outbreak management strongly depends on the commitment of the public to cooperate with outbreak investigation. The public must be willing to provide information, share specimens and temporary constrain liberty and privacy for the benefit of public's health at large [10]. This commitment of the public is not self-evident and can easily be hampered by careless use of the voluntary information provided by individuals, especially because in many (if not all) cases it will be unfeasible to obtain consent from all individuals involved in an outbreak [3, 10-13].

Ethical reflection on the use of typing techniques is therefore important. Professionals however, may perceive ethical reflection as complex and time consuming. The aim of this study was to articulate, explore and unravel the various ethical perspectives on the morally complex situation of using molecular typing methods in outbreak management. This in order to help professionals to develop a new and richer way of dealing with the ethical dimensions of using molecular science in response to infectious disease outbreaks.

\section{METHODS}

\section{Study design}

Empirical ethical approach based on dialogue between professionals.

\section{Participants}

For this study we invited professionals who use typing technique in their daily practice of outbreak management. In the Netherlands, three groups of professionals are mainly involved in outbreak management: (a) medical doctors (MD) specialised in infectious diseases control, (b) microbiologists/MD specialised in microbiology and hospital infections and (c) public health nurses specialised in infectious diseases. A total of 23 national representatives were invited and all accepted the invitation to participate. From September 2012 through February 2013 a total of three discussions were organised with, respectively, public health nurses specialised in infectious diseases control $(n=11)$, MD specialised in infectious diseases control $(n=7)$ and a mixed group consisting of national representatives of hospital MD specialised in microbiology and hospital infections $(n=3)$; microbiologists of the National Institute of Public Health and the Environment (RIVM) $(n=2)$; and a legal counsellor of RIVM $(n=1)$. All groups reached consensuses and consented with the presented summary.

\section{Group discussions}

General aim of the group discussions was to make explicit the shared moral understating of professionals that use molecular typing techniques in daily practice. The group discussions were semi-structured and moderated by a MD specialised in infectious disease control (B.R.) and an ethicists (C.C.). The discussions focussed on ethical considerations regarding the use of molecular typing techniques in an outbreak situation. Participants were asked to discuss what kinds of considerations would support a decision to use the technique and what considerations would give reason to abstain from using the technique. The participants were encouraged to work towards consensus.

In the topic list used for the discussions, we presented participants with five ethical questions: (1) Moral obligation to avoid spreading a disease: Can people be held responsible for transmitting infection? Why/Why not? (2) Ownership of pathogens: Do biological specimen, and the pathogen found in this specimen, in some way 'belong' to the individual they came from? Why/why not? (3) Informed consent: How to conceptualise the requirements for informed consent when using molecular techniques in outbreak management? (4) Return of results: Should information revealed by using the technique be returned to 
certain parties? Why/Why not? and (5) Legal perspective: Does introduction of molecular typing techniques bring forth new insights that influence underlying fundamental concepts of today's law? Why/Why not? [10]. These five ethical issues resulted from a previous theoretical analysis that included a literature study and an ethical debate on the use of the technique for outbreak management [10-13].

\section{Analysis}

The interviews were recorded and analysed through double coding by hand by two authors (B.R. and C. C.). Codes were clustered into (recurrent) themes. Themes and conclusions were approved by all interviewed groups. To make the themes more accessible for public health professionals, we rephrased the observed themes into interrogative sentences.

\section{RESULTS}

Is the possible harm for individuals in proportion with the expected public health benefits?

When outbreak managers were asked to explore the specific considerations that support and oppose the use of molecular microbial characterisation techniques, they first and foremost reflected on whether the possible harms for individuals were acceptable given expected public health benefits. This key question was approached by way of discussing four more specific ethical themes: (1) justification of governmental intervention, (2) responsibility to prevent infections, (3) scientific uncertainty and (4) legal consequences.

\section{Is governmental intervention justified in this case?}

Outbreak managers specifically questioned whether governmental intervention is justified in a given case, notably with regard to notification procedures and source and contact tracing, is justified in the first place. Respondents offered various criteria as to whether such government intervention is justified. It was argued that there must at least be a substantial public health threat in order to use molecular typing techniques in outbreak management, real time, without seeking explicit consent of all individuals involved. Also there should be a realistic expectation that deploying the techniques will help to mitigate the outbreak. Here, examples were given of an outbreak situation in which the use of the technique may provide information that will help to identify and disarm the source of an outbreak or of a situation in which the technique may provide insights on the scope of an outbreak, which will then help to determine the target population of a vaccination campaign. Finally, it was considered important to make sure that source and contact tracing would most likely not be successful without the use of molecular typing techniques.

\section{Do individuals have a responsibility to prevent infection?}

The participants proposed that the balance between harms and benefits tilts differently in different settings. It was argued, for instance, that certain individual professionals in specific regulated settings carry an explicit responsibility to prevent the spread of infectious diseases, such as medical professionals, chefs or food handlers. This was also argued for governments and companies in respect to product safety. In this context, the deployment of the technique may not only be useful in determining a source of infection, but also to exclude a relational pattern between two microbes, hereby discharging a potential source. In other, less-regulated settings, the balance tilts the other way and the protection of the individuals in terms of wellbeing, autonomy and liberty was considered more important. Here a responsibility to protect individuals from 'naming and shaming' was introduced.

\section{How to deal with the scientific uncertainty?}

A third major underlying theme addressed in the group discussions was scientific uncertainty. Outbreak managers elaborated extensively on the fact that conclusions based on molecular characterisation are always probabilistic in kind, leaving room for uncertainty. In practically all outbreaks, it was argued, it cannot be determined in advance whether the technique will in fact generate answers, what these answers will be and precisely how many uncertain factors those answers might contain. Even though the conclusions that typing techniques help reach can produce much more clarity on relational patterns than when using only epidemiological data, those conclusions remain probabilistic. Outbreak managers struggled with the fact that it cannot be determined in advance whether the use of molecular typing methods will in fact generate answers, and how much uncertainty those answers will have. It was proposed by some participants that an 
epidemiological investigation used to be the cornerstone of outbreak management, and that an epidemiological investigation reveals only circumstantial evidence. It was argued by others that whereas general public is well acquainted with the kind of uncertainty related to an epidemiological investigation, public expectations of molecular microbiological typing techniques cannot easily be tempered.

\section{What are the legal consequences?}

A last recurrent theme in the group discussions was the harm in terms of legal consequences for individuals. Participants evaluated the harm resulting from the use of the technique and whether this 'legal harm' is in proportion with the public health benefit. It was argued that the decision on using the technique was influenced by expectations of legal (liability) claims for individuals. Professionals seem to anticipate in their deliberating process on the possibility that information for source and contact tracing may be used at a later stage as evidence in a court case, which could have a negative effect on the commitment of the public to cooperate in future outbreak investigations.

\section{DISCUSSION}

The aim of this study was to articulate, explore and unravel the various ethical perspectives on the morally complex situation of using molecular typing methods in outbreak management. This in order to help future professionals to develop a new and richer way of dealing with the ethical dimensions of using molecular science in response to infectious disease outbreaks.

The basic assumption in this study was that a deeper ethical understanding is accomplished when the perspective of practitioners is not only used as a source of reflection by the ethicists, but when they are actually involved in the process of reflection and analysis. Articulating and defining the shared moral understanding of professionals will help to identify the ethical issues at stake when molecular science is used in an outbreak.

\section{What this study adds}

We now indeed found that the shared ethical understanding of the professionals differs from the theoretical ethical debate. First, ownership of the biological specimens, a theme that is considered highly relevant in the theoretical ethical debates, appeared relatively unimportant in the ethical discussions of the practitioners. This consideration addresses the question of who owns a biological specimen isolated from an individual, regarding whether a biological specimen (such as tissue, blood or stool) and the pathogen found in this specimen, in some way 'belong' to the individual they came from [14]. Interestingly, ownership was considered of little importance: participants argued, in line with public health law, that, if governmental interference is justified, then ownership of pathogens or specimens is irrelevant.

Dealing with scientific uncertainty, in turn, was put forward by the professionals of this study and not identified as such in a previous theoretical analysis [10]. This issue of scientific uncertainty showed to be an import issue that greatly influenced the decision making process of the professionals working in the field of outbreak management. We observed that professionals struggle with the fact that it cannot be determined in advance whether the use of molecular typing methods will in fact generate answers, and how much uncertainty those answers will hold.

We also observed that the potential legal consequences of an outbreak investigation profoundly dominated the group discussions. This may in part be explained by a fear of legal claims. Indeed, literature shows that characterisation can be a powerful instrument in a court of law. Not only in the USA, where claims may be more common, but also in Canada and in the Netherlands examples can be found of molecular characterisation techniques becoming part of legal arguments [3, 11, 12]. It is not hard to imagine how information about transmission routes of specific delicate infectious disease such as HIV or Ebola, once open for public, can fuel public debate and harm the involved individuals.

These differences illustrate that there is a gap between a theoretical ethical debate and a practical ethical case deliberation of professionals.

\section{Limitations of the study}

This study reflects the ethical deliberations of a selected group of professionals in the Netherlands; other stakeholders such as patients, contacts or other involved parties did not participate. Arguably, what is considered morally permissible will also be sensitive to the cultural and social context and hence be different outside the Netherlands. On the other hand, the values that are implied here are broadly accepted in modern medical and public health ethics [15-18]. 
It is important to note that the aim of this study was to describe how health care professionals actually reason ethically, this in contrast to exploring 'what professionals ought to do'. With this we adhere to the hermeneutic ethical perspective that theoretical ethical knowledge will ultimately derive its meaning and interpretation in the light of and application to practice. We think that articulating the shared moral understanding of using molecular science in response to infectious disease outbreaks will create a deeper and richer ethical understanding than theoretical ethical reflection on its own [19-21].

We found that the professionals in this project primarily questioned whether the potential harm the information may generate for involved individuals is in proportion with the public health benefit. This is not new nor surprising since this question represents the fundamental core of public health ethics: how to balance the interest of an individual with the interest of the public at large? Professional boundaries for what is prohibited, obligatory or permissible in outbreak management reflect this fundamental ethical question. The introduction and use of molecular typing techniques neither changes this fundamental question nor shifts the professional boundaries. It does, however, complicate the ethical deliberation within these boundaries.

\section{CONCLUSION}

Molecular typing methods can reveal chains in transmission pathways. These new chains all represent individual stakeholders whose interests need to be taken into consideration. This makes it important to not only reflect on the technical challenges related to using typing techniques in outbreak management but also include the ethical challenges. The themes that we found here and that we rephrased into questions, may help to articulate the ethical dimensions of using the technique in a future outbreak situations. More research is needed on how to bridge the gap between the theoretical ethical debate and the practical ethical case deliberations of professionals.

\section{ACKNOWLEDGEMENTS}

This work is funded by the Dutch Ministry of Health Welfare and Sport, RIVM, through the regional support fund for reinforcement of infectious disease control. The authors thank all professionals for their kind participation in the group discussions.

\section{DECLARATION OF INTEREST}

None.

\section{REFERENCES}

1. Houpikian P, Raoult D. Traditional and molecular techniques for the study of emerging bacterial diseases: one laboratory's perspective. Emerging Infectious Diseases 2002; 8: 122-131.

2. Sabat AJ, et al. Overview of molecular typing methods for outbreak detection and epidemiological surveillance. Euro Surveillance 2013; 18: 20380.

3. Millar M. Moral permissibility and responsibility for infection. Public Health Ethics 2012; 5: 314-317.

4. Van Duynhoven YT, et al. A prolonged outbreak of Salmonella Typhimurium infection related to an uncommon vehicle: hard cheese made from raw milk. Epidemiology and Infection 2009; 137: 1548-1557.

5. Barrabeig I, et al. Outbreak of Legionnaires' disease associated with a supermarket mist machine. Epidemiology and Infection 2010; 138: 1823-1828.

6. Stene-Johansen K, et al. An outbreak of hepatitis A among homosexuals linked to a family outbreak. Epidemiology and Infection 2002; 129: 113-117.

7. Centers for Disease, Control and Prevention. Escherichia coli $\mathrm{O} 157: \mathrm{H} 7$ infection associated with drinking raw milk-Washington and Oregon, November-December 2005. Morbidity and mortality weekly report 2007; 56: 165-167.

8. Buchholz U. et al. German outbreak of Escherichia coli O104:H4 associated with sprouts. New England Journal of Medicine 2011; 365: 1763-1770.

9. Friesema I, et al. Large outbreak of Salmonella Thompson related to smoked salmon in the Netherlands, August to December 2012. Euro Surveillance 2014; 19.

10. Rump B, et al. The need for ethical reflection on the use of molecular microbial characterisation in outbreak management. Euro Surveillance 2013; 18: 20384.

11. Bubela T, Yanow S. Molecular typing technology: a legal perspective. Public Health Ethics 2012; 5: 317-320.

12. Fanoy E, De Neeling A. Molecular typing: use with care. Public Health Ethics 2012; 5: 313-314.

13. Rump BO, Woonink F. Ethical questions concerning the use of molecular typing techniques in the control of infectious diseases. Public Health Ethics 2012; 5: 311-320.

14. Hawkins A, O'Doherty K. "Who owns your poop?": insights regarding the intersection of human microbiome research and the ELSI aspects of biobanking and related studies. BMC Medical Genomics 2011; 4: 72.

15. Beauchamp TL, Childress JF. Principles of Biomedical Ethics. Oxford University Press, 2001.

16. Lee LM, Heilig CM, White A. Ethical justification for conducting public health surveillance without patient consent. American Journal of Public Health 2012; 102: $38-44$.

17. Schroder-Back $\mathbf{P}$, et al. Teaching seven principles for public health ethics: towards a curriculum for a short 
course on ethics in public health programmes. $B M C$ Medical Ethics 2014; 15: 73.

18. Verweij M, Dawson A. Public Health Ethics, The International Encyclopedia of Ethics. Blackwell Publishing Ltd, 2013. 4220-4230 (DOI: 10.1002/ 9781444367072.wbiee699).

19. Salloch S, et al. The normative background of empirical-ethical research: first steps towards a transparent and reasoned approach in the selection of an ethical theory. BMC Medical Ethics 2015; 16: $1-9$.

20. Widdershoven G, Abma T, Molewijk B. Empirical ethics as dialogical practice. Bioethics 2009; 23: 236-248.

21. Frith L. Symbiotic empirical ethics: a practical methodology. Bioethics 2012; 26: 198-206. 